\title{
Zur Rolle der Lehrperson beim kooperativen Lernen
}

\section{Christine Pauli, Kurt Reusser}

Trotz des Vorliegens einer umfangreichen Forschungsliteratur zum kooperativen und selbständigen Lernen gibt es bislang nur wenige Publikationen zur Rolle und Wirksamkeit von Lehrpersonen bei Partner- und Gruppenarbeiten bzw. bei der Initiierung und Begleitung von schüleraktiven Lerndialogen. Bekannt ist dagegen, dass längst nicht alle in kooperativen Arrangements stattfindenden Lerndialoge und Interaktionen jene Qualitätsmerkmale aufweisen, von denen Pädagogen und Lehrpersonen gerne ausgehen. Auf der Basis vorliegender Erklärungshypothesen zum Nutzen kooperativer Lehr- und Lernformen wird im vorliegenden Text die Qualität von Lerndialogen für den multikriterialen Ertrag von Partner- und Gruppenarbeiten diskutiert. Anschliessend werden die Funktionen von Lehrpersonen im Hinblick auf die Sicherung dieser Qualität erörtert und es werden Folgerungen für die Ausrichtung der Forschung zur Lehrerrolle und zur Lernbegleitung beim dialogischen Lehren gezogen.

Menschen erwerben ihr Wissen und ihre Denkstrukturen nicht als einsame Sololerner, sondern im Rahmen sozialer Interaktionsgefüge und Austauschprozesse. Dies gilt nicht nur für das Lernen in Alltag und Beruf, sondern auch für die Schule, wo kooperatives Lernen eine zentrale Grundform und eine notwendige Ergänzung des Unterrichts im Klassenverband und in der Einzelarbeit darstellt. Wie eine mittlerweile grosse Zahl von Forschungsarbeiten belegt (vgl. aktuelle Übersichten z.B. in Dillenbourg, 1999; O’Donnell und King, 1999; Topping und Ehly, 1998; Webb und Palincsar, 1996), vermag kooperatives Lernen, sofern in geeigneter Form durchgeführt, positive Effekte auf eine ganze Reihe von sozialen, affektiven, motivationalen und kognitiven Verhaltensmerkmalen auszuüben.

In diesem Text wird die Bezeichnung kooperatives Lernen sowohl für Gruppenals auch für Partnerarbeitsformen im schulischen Unterricht verwendet. ${ }^{1}$ Damit sind Lernarrangements gemeint, die eine synchrone und koordinierte, ko-konstruktive Aktivität der Teilnehmer/innen verlangen, um eine gemeinsame Lösung eines Problems oder ein gemeinsam geteiltes Verständnis einer Situation zu entwickeln. 


\section{Begründungsmuster des kooperativen Lernens und die (vernachlässigte) Rolle der Lehrpersonen}

Aus der umfangreichen Forschungsliteratur wissen wir einiges darüber, was sich in Partner- oder Gruppenarbeiten abspielen kann bzw. unter Gesichtspunkten der Lernproduktivität abspielen sollte. Bekannt ist auch, wie häufig kooperative Lernformen in der Unterrichtspraxis realisiert und welche Sozialformen von Schülern und Schülerinnen bevorzugt werden (Huber, 1987; Nuhn, 1995; Stebler und Reusser, 1996, 2000). Es finden sich aber vergleichsweise wenige Untersuchungen, die auch das Verhalten der Lehrperson beim kooperativen Lernen einbeziehen, insbesondere deren lernbegleitende und lernberatende Tätigkeit während Gruppen- oder Partnerarbeiten. Vergegenwärtigt man sich die in der Literatur auffindbaren Begründungsmuster für den Nutzen kooperativen Lernens wird das geringe Forschungsinteresse an der Lehrperson zum Teil verständlich.

Pädagogische Begründungen des kooperativen Lernens verweisen in der Regel auf dessen Potenzial im Hinblick auf die Förderung sozial-kognitiver Schlüsselqualifikationen wie Teamfähigkeit, selbständiges Lernen, Sozial- und Kommunikationskompetenz. Da lehrerzentrierter Klassenunterricht wenig geeignet scheint, solche Kompetenzen heranzubilden, werden Autonomiespielräume gefordert, in denen Schüler/innen selbständig, zielgerichtet und eigenverantwortlich handeln und die erwünschten Kompetenzen einüben können. Gruppenarbeit, verstanden als nicht von Lehrpersonen (an)geleitetes Lernen, wird als solcher Spielraum des selbständigen und solidarischen Handelns gesehen (vgl. z.B. Gudjons, 1993; E. Meyer, 1954/1996; H. Meyer, 1987).

Lernpsychologische Begründungen legen das Gewicht auf die besondere kognitive und motivationale Qualität der Lernprozesse in kooperativen Lernsituationen. Ausgehend von einem konstruktivistischen Verständnis von Lernprozessen wird der besondere Nutzen kooperativen Lernens in der Förderung einer vertieften, aktiven und interaktiven Auseinandersetzung mit Problemen und Gegenständen in einem sozialen Kontext gesehen (Brown, 1997; Cobb et al., 1993; Huber, 1987; O’Donnell und King, 1999; Vettiger, 1987). Auch in dieser Argumentation wird eine Abgrenzung vom traditionellen Klassenunterricht und der damit verbundenen Steuerung durch die Lehrperson vorgenommen; beides wird tendenziell mit rezeptiv-passivem Lernen assoziiert, während kooperatives Lernen als Chance für (inter-)aktives, zielgerichtetes - sozialgenetisches oder sozial-konstruktives - Lernen betrachtet wird.

Beiden Argumentationsmustern gemeinsam ist der sich auf normative und empirisch-psychologische Überlegungen stützende Gedanke, dass multikriterial lernwirksame Gruppen- und Partnerarbeit neben der Akzentverschiebung der Lerntätigkeiten hin zu mehr Selbststeuerung und Eigenverantwortung auch die zeitweilige Emanzipation der Lernenden von ihren Lehrkräften erfordert. Konsequenterweise hat sich die Forschung zum kooperativen Lernen vor allem auf die Untersuchung solcher lehrerunabhängiger Aktivitäten in Paaren oder Gruppen 
und deren Wirksamkeit im Hinblick auf kognitive, affektive, kommunikative, motivationale und metakognitive Lernergebnisse konzentriert, während die Lernhilfe- und Betreuungsfunktion der Lehrpersonen weitgehend ausgeklammert wurde. Das heisst, während das selbständige Kommunikations- und Lernverhalten der Schüler und seine Wirkungen unter den vielfältigsten Kooperationsbedingungen analysiert worden sind, blieb das Lehrerverhalten in den meisten Studien unbeachtet.

Diese Vernachlässigung der Lehrfunktionen in der Forschung zum kooperativen Lernen hat zur Folge, dass bisher nur wenige empirisch fundierte Empfehlungen zu den didaktischen Möglichkeiten, kooperatives Lernen zu unterstützen, vorliegen; mit Ausnahme einer Reihe von Arbeiten zu sozialen Skripts oder Werkzeugen für die Gruppen- oder Partnerarbeit, wie z.B. «reziprokes Lehren» oder «strukturierte Interaktion» (vgl. Palincsar und Brown, 1984; Herrenkohl et al., 1999; Huber, 1991; O’Donnell und Dansereau, 1992; King, 1994, 1999; Pauli, 1998).

Im Vergleich zur Forschungsliteratur finden sich in didaktischen Lehrbüchern mehr Hinweise zu den Aufgaben der Lehrperson im Gruppenunterricht. Allerdings beziehen sich diese vor allem auf organisatorische Aspekte bei der Vorbereitung und Auswertung von Partner- und Gruppenarbeiten (z.B. H. Meyer, 1987). Hinsichtlich der Funktionen der Lehrperson während der Gruppen- oder Partnerarbeit überwiegen eher globale Hinweise darauf, dass Gruppenunterricht ein verändertes Verständnis der Lehrerrolle erfordert - «Moderation» gemeinsamer Lernprozesse anstatt "Wissens- und Kompetenzvermittlung» -, was beinhaltet, dass die Lehrperson möglichst nicht in das Gruppengeschehen eingreifen und die Lernenden ihrer autonomen Tätigkeit überlassen soll (vgl. z.B. Denecke und Ritz, 1993; Gudjons, 1993; Krapf, 1994; H. Meyer, 1987). Aufgabe der Lehrperson ist danach vor allem die gezielte Beobachtung der Gruppenarbeit, was auch Unterstützung und Beratung einschliessen kann. Konkrete Hinweise zu Unterstützungsmassnahmen betreffen vor allem sozialpsychologische und pädagogische Aspekte der Zusammenarbeit (Gruppendynamik, Dominanz usw.; vgl. z.B. Gudjons, 1993; Vettiger, 1987), während die Möglichkeiten der prozessbezogenen Lernhilfe, Unterstützung und Beratung hinsichtlich fachlich-kognitiver und metakognitiver Aspekte der Zusammenarbeit kaum konkretisiert werden.

Wir gehen jedoch davon aus, dass gerade auch in dieser Hinsicht ein Unterstützungsbedarf besteht. Denn die Produktivität von Partner- und Gruppenarbeiten hängt wesentlich von entsprechenden Qualitätsmerkmalen der Lerndialoge $a b$, die in den spontanen Dialogen in kooperativen Lernsituationen oft genug nicht auftreten.

Im Folgenden gehen wir auf der Grundlage zweier Erklärungshypothesen zu den Lernvorteilen kooperativen Lernens zuerst auf die Bedeutung der Qualität des Schülergesprächs für den Ertrag von Partner- und Gruppenarbeit und sodann auf die Funktionen der Lehrperson im Hinblick auf die Förderung und Sicherung dieser Qualität wie auch der Qualität der gemeinsam erzeugten Ergebnisse ein. 
Auf dieser Basis plädieren wir für eine vermehrte Berücksichtigung der Lehrerrolle in der Forschung zum kooperativen Lernen.

\section{Interaktionsqualität und Lernerfolg beim kooperativen Lernen}

Empirische und theoretische Forschungsarbeiten im Bereich des kooperativen Lernens kommen gleichermassen zum Schluss, dass die diskursive Qualität der Lernerinteraktion in der kooperativen Lernsituation bedeutsam ist für den individuellen Lernertrag (Cohen, 1994; O’Donnell und King, 1999; Stebler, 1999), dies vor allem, wenn es um anspruchsvolle Probleme und Lernaktivitäten geht. Folgende Merkmale von Lerndialogen wurden als bedeutsam identifiziert: Das Gespräch soll sich auf den Lerngegenstand beziehen und auf die Erarbeitung eines gemeinsam geteilten Verständnisses der Aufgabe (der Situation, des Problems) ausgerichtet sein; alle Kooperationspartner/innen sollen aktiv daran (und an der Lösung der Aufgabe) beteiligt sein, gegenseitig je auf die Beiträge und Fragen der Gesprächspartner/innen eingehen und diese weiterentwickeln, Sachverhalte gründlich erklären und Meinungsverschiedenheiten sachbezogen und argumentativ bearbeiten. In der Literatur werden solche Gesprächsmuster u.a. als «transaktiver Dialog» (Berkowitz und Gibbs, 1983), «connected discourse» (Hogan, Nastasi und Pressley, 1999), «exploratory talk» (Mercer, 1995) oder «KoKonstruktion» (Roschelle und Teasley, 1995) bezeichnet. Transaktive Dialoge schliessen eine Reihe von spezifischen soziokognitiven Aktivitäten ein, von welchen aufgrund unterschiedlicher Theorieansätze angenommen wird, dass sie für die Wirksamkeit des kooperativen Lernens insofern relevant sind, als die Zusammenarbeit den individuellen Lernzuwachs fördern soll.

Auf der Basis eines kognitiv-konstruktivistischen Entwicklungs- und Lernverständnisses im Sinne Piagets wird angenommen, dass die Konfrontation mit gegensätzlichen Standpunkten oder Sichtweisen zu kognitiven Konflikten führt und damit Anlass zur Weiterentwicklung bzw. Reorganisation der eigenen kognitiven Strukturen bildet (Piaget, 1947; Perret-Clermont, 1980). Doise und Mugny (1984) bezeichneten die durch Zusammenarbeit und Gedankenaustausch ausgelösten Konflikte als soziokognitive Konflikte. Die durch die Konfrontation mit neuer, nicht ohne weiteres assimilierbarer Information initiierten individuellen Aufbau- oder Umstrukturierungsprozesse kommen unter Umständen erst nach der Zusammenarbeit zum Tragen (vgl. Foot und Howe, 1998). Die Annahme, dass die Auseinandersetzung mit Gegenpositionen bei der Zusammenarbeit vergleichbar kompetenter Individuen zu individuellem Lernfortschritt führen kann, wird durch eine Reihe empirischer Untersuchungen gestützt (vgl. zusammenfassend De Lisi und Golbeck, 1999). Die Wirkung hängt jedoch nicht so sehr vom blossen Auftreten von Kontroversen zwischen den Teilnehmenden ab, sondern von der Qualität der Bearbeitung dieser Kontroversen. 
Aus der Sicht eines sozial erweiterten Konstruktivismus bzw. eines soziokulturalistischen Verständnisses kognitiver Entwicklung vor allem in der Tradition Vygotskys ist es das sozial konstruierte, gemeinsam geteilte Verständnis eines Problems oder einer Situation, das den Lernzuwachs ermöglicht. Wenn sich Individuen auf eine Lösungsstrategie oder eine gemeinsame Interpretation einer Sache einigen wollen, müssen sie das gemeinsame Verständnis aufgrund ihres unterschiedlichen Vorwissens und ihrer unterschiedlichen Perspektiven auf den Gegenstand aushandeln. Die sozial konstruierte, gemeinsam geteilte Sichtweise wird durch den Prozess des Aushandelns differenzierter und reichhaltiger als das je individuelle Vorverständnis. Lernfortschritt stellt sich dar als Teilhabe an diesem sozial konstruierten und sprachlich vermittelten Verständnis (Rogoff, 1990). Gemäss der soziokulturalistischen Auffassung kognitiver Entwicklung als Partizipation am Leben der Kultur eignen sich Kinder beim gemeinsamen Lösen von Aufgaben oder Problemen in der Zusammenarbeit mit kompetenteren Partnern (älteren Kindern, Erwachsenen) kulturell relevante Konzepte, Denkweisen, kognitive und metakognitive Strategien an, indem sie die zuerst im sozialen Verband gemeinsam ausgeführten und sprachlich vermittelten Vorgehensweisen und Konzeptbedeutungen allmählich verinnerlichen (Vygotsky, 1978). Dies unter der Voraussetzung, dass sowohl die Aufgaben als auch die Anleitung und Lernbegleitung durch kompetentere Personen optimal auf das Entwicklungspotenzial abgestimmt sind, d.h. in der «Zone der nächsten Entwicklung» erfolgen. Empirische Untersuchungen in dieser Forschungstradition konzentrierten sich anfänglich vor allem auf asymmetrische Lerndialoge (Erwachsene mit Kindern, Kinder mit unterschiedlichem Wissensstand; vgl. Rogoff, 1990). Mittlerweile wird auf dieser theoretischen Grundlage zunehmend auch die "gleichberechtigte» Interaktion von Lernpartnern mit vergleichbarer Kompetenz beim gemeinsamen Problemlösen analysiert und dokumentiert (vgl. zusammenfassend Hogan und Tudge, 1999), wobei vielfach auch auf Erkenntnisse der Ethnomethodologie und Konversationsanalyse zurück gegriffen wird. Dabei zeigt sich, dass auch vergleichbar kompetente Partner/innen sich in der Zusammenarbeit aufgrund ihrer unterschiedlichen Perspektiven und ihres unterschiedlichen Vorwissens wechselseitig unterstützen können. Aus theoretischer Sicht müsste allerdings die Zusammenarbeit mit einem kompetenteren Partner tendenziell wirksamer sein - was durch einige empirische Untersuchungen auch bestätigt wird (Hogan und Tudge, 1999) - oder es müsste zusätzliche Unterstützung oder Anleitung in anderer Form verfügbar sein, um Begriffe und Prozeduren, die als kulturelle Konstruktionen verstanden werden, zu erwerben.

Auch aus soziokulturalistischer Sicht spielt die Qualität der Interaktion eine wesentliche Rolle für den Ertrag der Zusammenarbeit. Wichtig ist insbesondere, dass alle (d.h. auch die weniger kompetenten) Partner aktiv an der Lösung der Aufgabe beteiligt sind und sich um die Aushandlung eines gemeinsam geteilten Verständnisses der Aufgabe bemühen. Wichtig ist ferner, dass stärkere Gruppenmitglieder ihre Überlegungen, z.B. bei Entscheidungen im Verlauf des Pro- 
blemlösens, verbalisieren und ihre kognitiven und metakognitiven Strategien explizit machen.

Insgesamt sprechen die Erklärungshypothesen beider theoretischer Traditionen ebenso wie empirische Studien dafür, dass sich erfolgreiche Gruppen- oder Partnerarbeiten, insofern sie auch kognitives und metakognitives Lernen fördern sollen, dadurch auszeichnen, dass alle Teilnehmenden sich aktiv und ko-konstruktiv an der gemeinsamen Lösung der Lernaufgabe(n) beteiligen, dass in einem qualitativ hochstehenden (transaktiven) Dialog ein gemeinsames Verständnis der Aufgabe und ihrer Lösung entwickelt wird, wobei Standpunkte argumentativ begründet, differierende und kontroverse Sichtweisen konstruktiv diskutiert, Strategien und Vorgehensweisen verbalisiert und Unklarheiten durch gegenseitige Hilfe und elaborierte Erklärungen beseitigt werden (vgl. Reusser, 2000). Dies ist vor allem dann bedeutsam, wenn es um fachlich anspruchsvolle Lernaktivitäten wie Begriffsbildung und Problemlösen und nicht um einfaches Üben und Repetieren im Sinne von Auswendiglernen oder Automatisieren geht. Sollen beim kooperativen Lernen nicht nur soziale, motivationale oder affektive, sondern auch kognitive und metakognitive Lernziele erreicht werden, muss somit dafür gesorgt werden, dass die Lerndialoge die entsprechenden Qualitätsmerkmale aufweisen.

\section{Aufgaben der Lehrperson beim kooperativen Lernen}

Beobachtungen des Interaktionsgeschehens in Gruppen- oder Partnerarbeiten zeigen indessen, dass die spontanen Dialoge der Lernenden sowohl inhaltlich als auch hinsichtlich der Gesprächsmuster oft nicht das erforderliche Qualitätsniveau erreichen. In empirischen Untersuchungen wurde festgestellt (z.B.: Cohen, 1994; Herrenkohl et al., 1999; Hogan, Nastasi und Pressley, 1999; Meloth und Deering, 1999; Pauli, 1998), dass

- viele Lernende vor allem darüber diskutieren, wie sie die Aufgabe möglichst schnell und einfach erledigen können, ohne sich inhaltlich damit beschäftigen zu müssen;

- sich das Gespräch häufig auf oberflächliche Aspekte eines Problems beschränkt, ohne die nötige Verstehenstiefe zu erreichen;

- Meinungsverschiedenheiten lediglich durch soziales Aushandeln und nicht durch sachbezogenes Argumentieren gelöst werden;

- Schüler/innen eher selten spontan relevante Fragen stellen, argumentieren und erklären, sondern die Kommunikation auf den Austausch von knappen Aufforderungen und einzelne Wortfragmente beschränken;

- schwächere Teilnehmer/innen sich nicht aktiv am Dialog beteiligen.

Lehrpersonen, die Partner- und Gruppenarbeiten durchführen, kommt deshalb die Aufgabe zu, dafür zu sorgen, dass die Qualität der Lerndialoge beim kooperativen Lernen in möglichst vielen Gruppen oder Paaren ein möglichst hohes Niveau erreicht, und dies sowohl hinsichtlich der soziokognitiven Gesprächskultur 
als auch hinsichtlich der fachlichen Bearbeitung der Aufgabe. Daraus ergeben sich eine Reihe von Aufgaben und Rollen der Lehrperson beim kooperativen Lernen. Zunächst gilt es kooperative Lernsituationen zu gestalten, welche eine qualitativ befriedigende Schülerzusammenarbeit fordern und ermöglichen - die Rolle der Lehrperson ist hier jene einer Choreographin und Designerin. Sodann werden Schüler/innen eher anspruchsvolle Dialoge führen und fachadäquat argumentieren, wenn sie Gelegenheit haben, das entsprechende Verhalten zu beobachten und einzuüben. Die Lehrperson ist daher auch Verhaltensmodell für kooperatives Lernen und Problemlösen. Während der Gruppen- oder Partnerarbeit ist sie zudem auch Lerncoach oder Beraterin, welche die Gruppen oder Paare bei Bedarf unterstützt. Als Expertin für den betreffenden Lerngegenstand trägt die Lehrperson überdies die Verantwortung für die sachliche Korrektheit der in den Paaren oder Gruppen entwickelten Problemlösungen und Konzepte. Und schliesslich hat sie als Moderatorin und Managerin des Lerngeschehens für den möglichst reibungslosen organisatorischen Ablauf der Lernaktivitäten sowie für die klassenöffentliche Integration und Sicherung der Ergebnisse von Gruppen- oder Partnerarbeiten zu sorgen. Im Folgenden sollen diese Funktionen erläutert werden.

\section{Die Lehrperson als Designerin kooperativer}

und dialogischer Lernsituationen

Wie produktiv sich die Zusammenarbeit in der Gruppen- oder Partnerarbeit gestaltet, hängt vom Zusammenspiel verschiedener Komponenten einer kooperativen Lernsituation ab. Designmerkmale kooperativer Lernsituationen, deren Einfluss auf die Qualität der Lernergebnisse und/oder der Lernerinteraktion (Lerndialoge) empirisch untersucht wurde, betreffen die Aufgabenstellung, Merkmale der Lernenden sowie die verfügbaren motivationalen Anreize (vgl. zusammenfassend Stebler, 1999; Webb und Palincsar; 1996).

In Bezug auf Merkmale der Lernenden und der Gruppenzusammensetzung wurde vor allem der Einfluss des Vorwissens, des Geschlechts und bestimmter Persönlichkeitsmerkmale der Lernenden sowie der Einfluss der sozialen Beziehungen in der Gruppe untersucht. Erwartungsgemäss zeigt sich, dass nicht alle Lernenden gleichermassen von kooperativem Lernen profitieren und dass die $\mathrm{Zu}-$ sammensetzung der Gruppe - z.B. homogen vs. heterogen in Bezug auf das Vorwissen oder die kognitiven Fähigkeiten - einen Einfluss auf den Lernertrag hat, wobei die günstigste Zusammensetzung vermutlich auch vom jeweiligen Lernziel der Gruppen- oder Partnerarbeit abhängt (vgl. Stebler, 1999). Auch die sozialen Beziehungen und der akademische Status sowie das Geschlecht der Partner/innen spielen eine Rolle. So sind Lernende mit höherem akademischem Status in Gruppenarbeiten aktiver und haben mehr Einfluss auf die Aufgabenlösung, und die Qualität der Zusammenarbeit ist in der Regel zwischen Lernenden des gleichen Geschlechts und zwischen befreundeten Lernenden besser als in geschlechtsgemischten Gruppen und zwischen nicht befreundeten Lernenden. 
In Bezug auf die motivationalen Anreize erwiesen sich vor allem zwei Merkmale der kooperativen Situation als bedeutsam. Zum einen muss Zielinterdependenz bestehen, d.h. die Lernenden müssen ein Ziel erkennen, das am besten durch gemeinsame Anstrengung und gegenseitige Unterstützung zu erreichen ist. Zum andern sollten sich alle Gruppen- oder Paarmitglieder je persönlich verantwortlich fühlen für die Zielerreichung (Johnson und Johnson, 1992b; Slavin, 1993). Offen ist, wie solche Anreizstrukturen geschaffen werden können. Während einige Forscher/innen davon ausgehen, dass dies nur über äussere Anreize in Form von Gruppenbelohnungen geschehen kann (Slavin, 1993), sind andere der Meinung, dass eine gute Vorbereitung der Lernenden, interessante, ansprechende Aufgaben und die Gruppenkohäsion ebenso geeignet sind, um die Lernenden zu engagierter Zusammenarbeit zu motivieren (Cohen, 1994). In die bewusste Gestaltung der motivationalen Anreizstruktur muss auch die Bewertung der Leistungen bei Gruppen- oder Partnerarbeiten einbezogen werden.

In Bezug auf die Aufgaben zeigen Untersuchungen, dass eine sachbezogene Auseinandersetzung im Dialog vor allem dann geführt wird, wenn die Aufgabe anspruchsvoll und komplex ist, mehrere Lösungswege zulässt, sich jedoch nicht in einzelne Teilaufgaben aufteilen lässt, die mit Vorteil von den einzelnen Mitgliedern individuell bearbeitet werden (Cohen, 1994). Wodurch genau sich geeignete Lernaufgaben auszeichnen, ist jedoch nicht ausreichend untersucht. Neben der Art der Aufgabe selbst beeinflusst auch die Klarheit und Verständlichkeit der Aufgabenstellung bzw. des Arbeitsauftrags die Qualität und den Ertrag von Gruppenarbeiten (Fürst, 1999). Gezielte Instruktion der Lernenden vor der Gruppenarbeit hat sich in verschiedenen Untersuchungen als wirksames Mittel zur Steigerung der Qualität von Lerndialogen erwiesen. Dazu gehört, dass den Lernenden das Ziel der Aufgabe klar gemacht wird und dass sie auf geeignete kognitive und metakognitive Strategien hingewiesen werden, aber auch darauf, welche Form der Zusammenarbeit bei der gegebenen Aufgabe besonders vorteilhaft ist (Cooper, 1999; Meloth und Deering, 1999).

Insgesamt sind die Forschungsergebnisse zu den Rahmenbedingungen kooperativen Lernens vielschichtig und nicht leicht zu interpretieren. Dies auch deshalb, weil zuwenig über das Zusammenspiel einzelner Komponenten, die sich vermutlich teilweise gegenseitig kompensieren oder aufheben, bekannt ist (Dillenbourg, 1999; Renkl und Mandl, 1995; Webb und Palincsar, 1996). Zudem ist anzunehmen, dass unterschiedliche Lernziele auch zum Teil unterschiedliche Anforderungen an die Gestaltung kooperativer Lernsituationen stellen. Vor diesem Hintergrund erweist sich die Aufgabe der Lehrperson, ertragreiche kooperative Lernsituationen zu entwerfen und im Klassenzimmer zu gestalten, als höchst komplex und anspruchsvoll, und Lehrpersonen müssen davon ausgehen, dass es immer auch Gruppen oder Paare gibt, bei denen - aus unterschiedlichen Gründen - keine optimale Zusammenarbeit zustande kommt.

Hilfreicher im Hinblick auf konkrete Handlungsempfehlungen präsentiert sich der Forschungsstand in Bezug auf die Wirkung sozialer Werkzeuge oder Un- 
terstützungsmassnahmen auf das kooperative Lernen. In den letzten Jahren wurden verschiedene Strukturierungsmassnahmen und Lernhilfen entwickelt und systematisch überprüft, mit denen kooperative Lernsituationen fach- und zielbezogen angereichert werden können. Im Vergleich mit den Prozessen und Ergebnissen unangeleiteter oder unstrukturierter Zusammenarbeit erwiesen sich solche Lernhilfen als wirksam, und zwar sowohl in Bezug auf den Gesprächsinhalt (z.B. Verstehenstiefe) als auch in Bezug auf die soziokognitive Qualität von Dialogen. Eine wirksame Form der Unterstützung besteht in der Strukturierung der Interaktion durch «Skripts», die den Lernenden bestimmte Rollen vorgeben, die mit der Ausführung bestimmter Strategien verbunden sind. Bekannte Skripts, deren Wirksamkeit belegt ist, sind die "Konstruktive Kontroverse» (Johnson und Johnson, 1992a), das "Reziproke Lehren» (Palincsar und Brown, 1984) und die "Scripted Cooperation» (O’Donnell und Dansereau, 1992). Weniger klar ist, inwieweit stark strukturierende Skripts, die vor allem für das Lesen bzw. den textbasierten Wissenserwerb entworfen wurden, auch auf andere, offenere Lernsituationen, z.B. problemlösende oder explorative Lernaktivitäten übertragbar sind. So wird befürchtet, dass die stark vorstrukturierten Abläufe einzelner Skripts die erwünschten kognitiven und metakognitiven Aktivitäten möglicherweise geradezu einschränken (Cohen, 1994; Rosenshine und Meister, 1994). Flexibel nutzbare Ressourcen und "Denk-Werkzeuge», die strategisches Vorgehen anregen, ohne die Lernenden einem starren Ablauf des Vorgehens zu unterwerfen, scheinen hier sinnvoller zu sein und haben sich für verschiedene Fächer und Lernaufgaben als wirksam erwiesen, und zwar sowohl hinsichtlich des fachlichen Lernens wie auch hinsichtlich der Ausbildung kognitiver und metakognitiver Strategien (Herrenkohl et al., 1999; King 1999). Auch computerunterstützte Lernwerkzeuge für Gruppen- oder Partnerarbeit bieten vielfache Möglichkeiten flexibler Unterstützung (Dillenbourg, 1999; Reusser, 1993). In einer Untersuchung zum paarweisen Lösen von Textaufgaben stellten wir fest, dass die Verfügbarkeit einer computerunterstützten Lernhilfe, die in erster Linie ein Repräsentationsformat zur Verfügung stellte und den Problemlösungsprozess minimal strukturierte, die Lerndialoge von Fünftklässlern im Vergleich zur unangeleiteten Zusammenarbeit qualitativ und quantitativ wesentlich veränderte (Pauli, 1998). Geeignete Repräsentationsformate helfen den Lernenden, sich über schwierige und abstrakte Sachverhalte besser zu verständigen und fördern so den sprachlichen Austausch im Dialog. Ferner kann durch minimale Strukturierungen (z.B. Situationen mit Aufforderungscharakter) adäquates strategisches und argumentatives Verhalten modelliert oder angeregt werden, beispielsweise dadurch, dass Entscheidungssituationen geschaffen werden, die von den Lernenden verlangen, sich über strategische und metakognitive Aktivitäten zu verständigen.

Insgesamt wurde im Bereich der Unterstützung kooperativen Lernens durch Lernhilfen und Strukturierungen in jüngster Zeit eine ganze Reihe von Arbeiten publiziert, die zeigen, dass es möglich ist, die Qualität der Schülerzusammenar- 
beit auch mit wenig Aufwand durch geeignetes didaktisches Design und durch das Verfügbarmachen von Lernwerkzeugen wirksam zu unterstützen.

\section{Die Lehrperson als Verhaltensmodell und Lerngerüst}

Gruppen- und Partnerarbeiten werden zu Recht als Lerngelegenheiten angesehen, Strategien des Problemlösens sowie fachspezifische Formen der Interaktion, des Austauschs, des Formulierens und Argumentierens einzuüben (z.B. «scientific literacy»; Lemke, 1990). Wie oben dargelegt wurde, kann jedoch nicht davon ausgegangen werden, dass adäquates, zielgerichtetes Argumentieren, überhaupt alle effektiven und expertenhaften Formen des Austauschs und der Interaktion, spontan auftreten oder erworben werden. Das fragend-entwickelnde Lehrgespräch, das im Klassenunterricht meistens dominiert, ist nur bedingt geeignet, die Lernenden auf selbstgesteuerte Lerndialoge in der Gruppenarbeit vorzubereiten. Lehrgespräche sind in der Regel stark von der Lehrperson gesteuert und folgen einem klassischen Gesprächsmuster, das als Abfolge von IRE-Sequenzen (Initiation - Response Evaluation) beschrieben wurde (Mehan, 1979). Die Rolle der Lernenden ist hier vor allem jene der Antwortenden, während praktisch alle Initiativen und Steuerungsimpulse zum Fortgang des Gesprächs - und meistens auch zur Entwicklung der fachlichen Problemlösung - von der Lehrperson ausgehen. Beim gemeinsamen und eigenständigen Problemlösen in Gruppen- oder Partnerarbeit müssen die Lernenden im Dialog die aktive Rolle selber übernehmen - eine Rolle, die ihnen neu ist, wenn sie nicht immer wieder Gelegenheit haben, zielorientiertes und fachlich richtiges Argumentieren zu beobachten und unter Anleitung zu praktizieren (Bauersfeld, 1995; Cooper, 1999). Klassendiskussionen, die bewusst als solche (und nicht als lehrerzentrierte klassische Lehrgespräche) gestaltet werden, können solche Gelegenheiten bieten (vgl. z.B. Herrenkohl et al., 1999; Lampert, 1990). Das Lernen in Kleingruppen selber bietet ebenfalls ideale Gelegenheiten für die Lehrperson, entsprechende Dialogmuster im Gruppengespräch zu modellieren und als Lerngerüst (scaffolding) - mittels reflexionsbezogener Metakommunikation - anzuleiten und zu unterstützen (Cobb, Wood und Yackel, 1993; Cooper, 1999; Hogan, Nastasi und Pressley, 1999; Palincsar und Brown, 1984).

Der Aufbau einer dem Fachinhalt gerecht werdenden Problemlösungs- und Interaktionskultur, die es ermöglicht, dass Gruppen- und Partnerarbeiten zu Gelegenheiten zum zielgerichteten gemeinsamen Problemlösen und zur fachgerechten Diskussion werden, ist ein längerfristiger Prozess, der die bewusste Kultivierung von Unterrichtsgesprächen im Sinne der Modellierung adäquater Dialog- und Argumentationsmuster sowohl im Klassen- wie auch im Gruppenunterricht erfordert. Das heisst, was hier über die modellierenden und lernunterstützenden Funktionen von Lehrpersonen beim kooperativen Lernen gesagt wurde, gilt gleichermassen auch für die Modellierungsfunktion beim Führen von Lehr-Lerndialogen im Ganzklassenunterricht. 


\section{Die Lehrperson als adaptive Lernberaterin \\ während der Gruppenarbeit}

Der Frage, welche Funktion der Lehrperson während der Gruppen- oder Partnerarbeit ihrer Schüler/innen zukommt, ist von der Forschung zum kooperativen Lernen bislang weit weniger Aufmerksamkeit geschenkt worden als der Frage, wie kooperative Lernsituationen von den äusseren Bedingungen her gestaltet und mit Lernhilfen angereichert werden können. Selbst wenn Lernhilfen verfügbar sind, zeigen Beobachtungen, dass nicht alle Lernenden gleichermassen fähig sind, sie auch produktiv zu nutzen (Pauli, 1998), so dass eine zusätzliche, adaptive Unterstützung und Lernhilfe durch die Lehrperson erforderlich ist (Littleton und Häkkinen, 1999).

Die wenigen Untersuchungen, die bisher dazu vorliegen, zeigen, dass die Beratungs- oder Coachingfunktion beim kooperativen Lernen hohe Anforderungen an die Lehrpersonen stellt. Was das Eingreifverhalten von Lehrpersonen während Gruppenarbeiten anlangt, zeigen mikroanalytische Untersuchungen der Forschungsgruppe um Dann, Diegritz und Rosenbusch (Fürst, 1999) auf der Basis von Beobachtungen des Verhaltens von zehn Lehrpersonen in über 40 Gruppenarbeiten, dass der Adaptivität der Lehrerinterventionen eine zentrale Rolle mit Bezug auf die Wirksamkeit des Eingreifens zukommt. Wichtig scheint, dass sich die Lehrperson zuerst über den aktuellen Stand des Gruppengesprächs informiert, um entsprechend dem Verständnis und allfälligen Schwierigkeiten der Schüler/innen situationsbezogen eingreifen zu können. Wie die Studie zeigt, folgte auf Interventionen mit geringem Situationsbezug häufig eine Verschlechterung der inhaltlichen Progression in den Gruppengesprächen (ebd., S. 138 ff.). Die Beratung hatte somit negative Wirkungen. Unglücklicherweise scheinen Lehrpersonen dazu zu tendieren, sich wenig situationssensibel zu verhalten, nämlich von Gruppe zu Gruppe zu gehen und einzugreifen, ohne sich vorgängig genügend orientiert zu haben - ein Verhalten, das auch in anderen Untersuchungen beobachtet wurde (vgl. Meloth und Deering, 1999). Aufgrund ihrer Ergebnisse plädieren Diegritz et al. (1999) dafür, dass Lehrpersonen während der Gruppenarbeit «möglichst wenig (d.h. selten, kurz, am besten gar nicht) intervenieren» sollten (S. 346). Falls Interventionen nicht vermeidbar sind, sollen sie zurückhaltend, situationsbezogen und präzis, aber möglichst nicht lenkend erfolgen, um zu vermeiden, dass sich das Gespräch nach dem vertrauten Interaktionsmuster des Frontalunterrichts entwickelt bzw. die intendierte Lernunterstützung zum «MiniFrontalunterricht» wird (ebd., S. 347).

Andererseits kommen Meloth und Deering (1999) aufgrund von Analysen von Lehrer-Schüler-Interaktionen während Gruppenarbeiten zum Schluss, dass auch längere Lehrerinterventionen während der Gruppenarbeit - sie sprechen von «mini-lessons» - weder die selbständige Zusammenarbeit noch die Qualität des Lerndialogs beeinträchtigen müssen. Effektive Lehrer-Schüler-Interaktionen beim kooperativen Lernen, welche die Zusammenarbeit und den sachbezogenen Lerndialog zu intensivieren vermögen, zeichnen sich dadurch aus, dass sie so- 
wohl inhaltliche Informationen im Hinblick auf die Lösung der Aufgabe ins Gespräch einbringen als auch Hilfen für die Zusammenarbeit in Gruppen vermitteln, wobei die Lehrperson selber wenig Fragen stellt, sondern direkte und präzise Anweisungen und Erklärungen gibt. Meloth und Deering kommen deshalb zum Schluss, dass die Wirkung von Lehrerinterventionen weniger von deren Häufigkeit oder zeitlicher Ausdehnung abhängt als von deren Qualität und Adaptivität.

Hogan, Nastasi und Pressley (1999) untersuchten die soziokognitiven Gesprächsmuster und die inhaltliche Qualität der Lerndialoge von vier Schülergruppen bei der Bearbeitung von anspruchsvollen, explorativen Lernaufgaben im projektartigen Unterricht, und zwar sowohl dann, wenn die Gruppen selbständig arbeiteten als auch dann, wenn die Lehrerin am Gruppengespräch teilnahm. Insgesamt waren lehrergeleitete Gruppendiskussionen erwartungsgemäss effizienter und wiesen ein höheres inhaltliches Niveau auf, während selbständige Diskussionen explorativer waren. Obwohl die Lehrerin viele Fragen stellte, unterschieden sich die lehrergeleiteten Gruppengespräche wesentlich von einem eigentlichen Lehrgespräch im Sinne oben erwähnter I-R-E-Sequenzen. Der Unterschied bestand vor allem darin, dass die Lehrerfragen Reaktionen auf Beiträge der Lernenden darstellten und nicht von der Lehrperson im voraus geplant waren. Weiter machen die Mikroanalysen deutlich, dass zwischen den selbständigen Lerndialogen der Gruppen erhebliche Qualitätsunterschiede auftraten, und sie zeigen, auf welche Weise es der Lehrperson gelang, die Gesprächsqualität der schwächeren Gruppen während ihrer Teilnahme wesentlich zu steigern. Bei Gruppen, die selbständig auf hohem Niveau diskutierten, führte die Anwesenheit der Lehrperson zu einer Abnahme der Komplexität der Argumente.

Alle drei Untersuchungen weisen auf die grosse Bedeutung der Adaptivität des Lehrerverhaltens bei der aktiven Unterstützung von Gruppenarbeiten hin. Es ist entscheidend, dass Lehrpersonen die Gruppen oder Paare sehr gut beobachten, damit sie in der Lage sind, gezielt und bezogen auf den aktuellen Gesprächsstand einzugreifen. Weitere Untersuchungen sind aber notwendig, um zu klären, wann, d.h. in welchen Situationen eine Intervention sinnvoll oder erforderlich ist, auch wenn die Lernenden selber keinen Bedarf anmelden, und wie allfällige negative Auswirkungen von Interventionen vermieden werden können.

\section{Die Lehrperson als Expertin für den Lerninhalt} Vor dem Hintergrund eines kognitiv-konstruktivistischen Verständnisses von Lernen wird kooperatives Lernen vor allem als gemeinsames Explorieren und Problemlösen im Rahmen mehr oder weniger «entdeckender» und sozial «offener» Formen des Unterrichts inszeniert, wobei der Dialog als entscheidendes Prozessmerkmal betrachtet wird. Doch selbst ein optimal transaktiver Dialog bietet noch keine Gewähr für sachbezogene Lernfortschritte. So können beispielsweise im Dialog auch Missverständnisse und Fehlkonzepte ko-konstruiert werden, und die 
Zusammenarbeit mit einem schwächeren Partner kann zur Regression des Stärkeren anstelle der Progression des Schwächeren führen (Tudge, 1989).

Aus soziokulturalistischer Sicht wird schulisches Lernen als Akkulturation in die Praxis verschiedener Disziplinen oder Fächer gedeutet (Herrenkohl et al., 1999). Fachlicher Wissenserwerb heisst demzufolge, mit den Tätigkeiten, Prinzipien und Begriffen vertraut zu werden, die für die Teilnahme an der kulturellen Praxis - zum Beispiel jener der Naturwissenschaften oder der Mathematik erforderlich sind. Gemeinsame Aktivitäten mit kompetenteren Partnern werden als Gelegenheiten verstanden, kulturrelevante Begriffe, Repräsentations- und Denkformen zu erwerben. Obwohl die aktive und konstruktive Auseinandersetzung von gleichermassen kompetenten Partnern beim Explorieren, Experimentieren oder Problemlösen zu einer Vertiefung des Verständnisses und sogar zur Entdeckung von Gesetzmässigkeiten oder Prinzipien führen kann, muss es dennoch als äusserst unwahrscheinlich gelten, dass sich kooperativ und selbständig Lernende - auch bei Voraussetzung einer «idealen» Dialogkultur - das Wissen und Können fachinhaltlicher Denkkulturen selbständig und ohne signifikante Unterstützung durch kompetente Vertreter dieser Kultur aneignen können. So werden die Lernenden spätestens dann Unterstützung brauchen, wenn es darum geht, ihre selbst gewonnenen Erkenntnisse auf Begriffe und dadurch in Einklang mit den geltenden Konventionen und Verfahren einer Fachdisziplin zu bringen (Edwards und Mercer, 1989). Im schulischen Unterricht hat die Lehrperson in Wahrnehmung ihrer Repräsentationsverantwortung für Kultursegmente (Fächer) die Rolle des kompetenten Partners, und damit der Hüterin von Standards beim gemeinsamen Lösen von Problemen, zu spielen. Dies, indem sie ihren Wissensund Könnensvorsprung in den Lerndialog einbringt und im Austausch mit den Lernenden die Ko-Konstruktion von Konzepten und fachspezifischen Argumentations-, Schluss- und Beweismustern unterstützt (Herrenkohl et al., 1999; Cooper, 1999; Hogan, Nastasi und Pressley, 1999).

Für das explorierende und problemlösende kooperative Lernen bedeutet dies, dass die von den Gruppen oder Paaren erzeugten Lösungen und Ideen auf der Basis fachlicher Gütestandards evaluiert und gegebenenfalls korrigiert werden müssen. Insofern dies nicht während der Gruppen- oder Partnerarbeit geschieht, muss es in Auswertungsphasen geschehen, die an Gruppen- oder Partneraktivitäten anschliessen und die mehr beinhalten als blosse Ergebnispräsentation oder Metainteraktion über die personale und soziale Befindlichkeit. Wie insbesondere die Arbeiten von Cobb und weiteren Autor/innen zeigen, ist es mit einem konsequenten Aufbau einer entsprechenden Lernkultur, dem Einüben fachspezifischer Denk- und Argumentationsfiguren und mit geschickter Gesprächsführung durch die Lehrperson schon bei jungen Schülern und Schülerinnen möglich, im Anschluss an kooperative Lernphasen die darin erzeugten Ideen und ausgehandelten Lösungen mit den Lernenden fachgerecht zu diskutieren, bis ein adäquates, gemeinsam geteiltes Verständnis einer Sache erreicht ist (Cobb, Wood und Yackel, 1993; Herrenkohl et al., 1999; Lampert, 1990). 
Die Lehrperson als Organisatorin und Moderatorin Kaum strittig ist ein letzter funktionaler Aspekt der Lehrerrolle, nämlich jener der Organisation und Moderation der Schüleraktivitäten. Dass das Gelingen von Gruppenarbeiten - wie überhaupt von Unterricht - wesentlich von organisatorischen Massnahmen abhängt, ist eine Binsenwahrheit (vgl. Helmke und Weinert, 1997). Nicht nur ermöglicht ein gutes gruppenunterrichtsbezogenes «Classroom Management» den reibungslosen Ablauf des Gruppenunterrichts und maximiert so die aufgabenbezogene Lernzeit («time on task»), sondern es trägt auch zum Wohlbefinden der Schüler und Schülerinnen beim kooperativen Lernen bei, z.B. wenn unnötiger Zeitdruck vermieden wird (Nuhn, 1995). Ein wesentliches Element der Planung kooperativen Lernens sind ebenfalls Überlegungen zu den Lernaktivitäten vor und nach der eigentlichen Gruppen- oder Partnerarbeit, also den Phasen des Arbeitsauftrags und der Auswertung. Wie bereits erwähnt, ist insbesondere bei problemlösend-entdeckenden Lernaktivitäten die Auswertungsphase ein zentrales Element des kooperativen Lernens und erfordert eine gezielte Vorbereitung und bewusste Gestaltung. Die Herausforderung besteht darin, eine Präsentation der Gruppenergebnisse so zu moderieren, dass keine Langeweile aufkommt und ein möglichst konstruktiver Austausch auch zwischen den Gruppen und Schülerpaaren stattfinden kann. In den Untersuchungen von Dann, Diegritz und Rosenbusch (1999) erwiesen sich vor allem die Sicherung des erzeugten Wissens und dessen vernetzende Integration in Zusammenhänge als jene Aktivitäten in der Auswertungsphase, die die Aufmerksamkeit der Schüler/innen am meisten zu fesseln vermochten.

\section{Die Rolle der Lehrperson und die Forschung zum kooperativen Lernen}

Unser Überblick über Aspekte der Lehrerrolle beim kooperativen Lernen sollte deutlich machen, dass der Lehrperson beim kooperativen Lernen eine grössere Bedeutung zukommt, als es aufgrund der bisherigen Forschungsliteratur den Anschein macht. Als Gestalterin von Lernsituationen und Entwickler von zusätzlichen Lernhilfen oder Stützmassnahmen, als Verhaltensmodell für fachspezifisches Problemlösen, für konstruktives Kooperieren und Diskutieren, als Organisatorin und Moderatorin von kooperativen Lernaktivitäten und als Experte der Fachdisziplin kann die Lehrperson auf verschiedenen Ebenen, zu verschiedenen Zeitpunkten und mit verschiedenen Mitteln den Erfolg von Gruppenund Partnerarbeit aktiv beeinflussen. Diese verschiedenen Lehrfunktionen in die Forschung zum kooperativen Lernen vermehrt einzubeziehen, erachten wir aus verschiedenen Gründen als wichtig. Zum einen liegen zu bestimmten Aspekten noch kaum empirische Daten vor, oder die vorliegenden Ergebnisse sind widersprüchlich. Zum andern fehlt es bisher vielfach an konkreten Emp- 
fehlungen, die Lehrpersonen helfen könnten, in ihrem Unterricht vermehrt qualitativ gute Gruppen- oder Partnerarbeiten durchzuführen.

Untersuchungen haben wiederholt ergeben, dass kooperatives Lernen im Unterricht wesentlich seltener praktiziert wird als Klassenunterricht und Einzelarbeit (Huber, 1987; Nuhn, 1995), zumal in Kernfächern wie z.B. Mathematik (Beaton et al., 1996; IAEP, 1992; Stebler und Reusser, 1996)², obwohl die neuere pädagogisch-psychologische Forschung eine Kombination verschiedener Lehrformen im Unterricht nahelegt (Weinert, 1997). Da Lehrpersonen primär affektive und soziale Ziele im Auge haben, wenn sie kooperatives Lernen durchführen (Meloth und Deering, 1999; Stebler und Reusser, 1996), könnte ihre Zurückhaltung gegenüber kooperativen Lernformen in der Befürchtung begründet sein, die angestrebten positiven Wirkungen des Gruppenunterrichts, wie z.B. Erziehung zur Selbständigkeit, das Einüben von Kooperationsfähigkeiten usw., könnten auf Kosten des fachlichen und kognitiven Lernens gehen (Huber, 1987; Maheady, 1998) ${ }^{3}$. So gaben beispielsweise in einer Umfrage zur Partnerarbeit von Nuhn (1995) mehr als 38 Prozent der Lehrpersonen an, dass ein Nachteil der Partnerarbeit in der Untätigkeit der leistungsschwachen Schüler bestehe. Lehrerinterviews der Forschungsgruppe um Dann, Diegritz und Rosenbusch (Dann et al., 1999) zeigen zudem, dass die Durchführung von Gruppenunterricht für die Lehrpersonen mit Entscheidungskonflikten verbunden ist, die sich vor allem im Dilemma «Eingreifen oder Nicht-Eingreifen» kristallisieren. Da Gruppenarbeit vor allem als Freiraum für autonome Tätigkeiten begriffen wird, erachten es die Lehrpersonen als wichtig, sich möglichst zurückzuhalten und die Schüler selbständig arbeiten zu lassen; gleichzeitig sehen sie sich aber auch veranlasst, kontrollierend einzugreifen (Von Hanffstengel, 1999). Angesichts der Tatsache, dass die Qualität der Lerndialoge bei Gruppenarbeiten im Schulalltag oft sehr mangelhaft ist, sind die Entscheidungsdilemmata der Lehrpersonen verständlich. Soll es beim kooperativen Lernen nicht zu einem Zielkonflikt zwischen sozial-affektiven Lernzielen einerseits und kognitiven Lernzielen andererseits kommen, müssten vermehrt Kenntnisse darüber erarbeitet werden, wie auch der kognitive Lernertrag bei Gruppen- und Partnerarbeiten gesichert werden kann, ohne das Ziel kognitiv anspruchsvollen selbständigen Arbeitens aufzugeben. Daraus wären dann auch praxisrelevante Empfehlungen für Lehrpersonen abzuleiten.

In Bezug auf die Gestaltung kooperativer Lernumgebungen sind die bisherigen Forschungsergebnisse insgesamt für die Unterrichtspraxis nicht sehr hilfreich. Auch wenn damit begonnen wurde, das Zusammenspiel einzelner Komponenten kooperativer Lernsituationen systematischer zu untersuchen (z.B. die Beziehung zwischen Merkmalen der Organisation, der Anreizstruktur und zusätzlichen personalen Unterstützungsmassnahmen), bleibt es schwierig, auf dieser Basis praxisrelevante Empfehlungen zu formulieren.

Anders sieht es in Bezug auf die Entwicklung und Erprobung didaktischer Design-Massnahmen oder Lernhilfen aus, mit deren Hilfe die Qualität von Lerndialogen und der Ergebnisse von Gruppen- oder Partnerarbeiten wesentlich 
verbessert werden kann. Einige dieser Unterstützungsmassnahmen - darunter die «konstruktive Kontroverse» und das «reziproke Lehren» - können mittlerweile auch im deutschsprachigen Raum als bekannte Verfahren kooperativen Lernens betrachtet werden (vgl. z.B. Huber, 1987, 1991; Aeschbacher, 1989; Gräsel und Gruber, 2000; Krapf, 1994). Insofern es sich bei diesen Hilfsmitteln um relativ stark strukturierende Lernarrangements handelt, wäre es wünschenswert, wenn vermehrt auch flexiblere didaktische Werkzeuge zur Unterstützung des kooperativen Lernens im deutschen Sprachraum zugänglich gemacht, kreativ weiterentwickelt und hinsichtlich ihrer Wirkung überprüft würden. Letzteres drängt sich auf, weil aus der Literatur bekannte Instrumente zum Teil auf bestimmte Fächer, Lernziele, Schulstufen und auch Lernkulturen ausgerichtet sind. Bestehende, in bestimmten Kontexten erprobte Arrangements könnten so Ausgangspunkte bilden für die Entwicklung und systematische Erprobung weiterer, auf bestimmte Fächer und/oder Stufen abgestimmter Stützmassnahmen im Rahmen von «Design-Experimenten» (Brown, 1992).

Unabhängig von der Anreicherung von kooperativen Lernsituationen mit zusätzlichen Ressourcen oder Stützmassnahmen, stellt sich weiterhin die Frage, welche Rolle Lehrpersonen während der Gruppen- oder Partnerarbeit zukommt, insbesondere die Frage, wann und wie Lehrpersonen in das Geschehen und in die Gespräche der Gruppen oder Paare eingreifen sollen. Ergebnisse einiger Untersuchungen weisen darauf hin, dass die Förderung der Selbständigkeit einerseits und steuerndes Eingreifen andererseits nicht zwingend in Widerspruch zueinander stehen müssen. So konnte gezeigt werden, dass wohl dosierte, adaptive Interventionen von Lehrpersonen zur Intensivierung der kognitiven Aktivitäten bei den Schüler/innen führen können (Cooper, 1999), dass Lernende solche Interventionen als hilfreich empfinden (ebd.), und dass die Interventionen nicht zu vermehrter Abhängigkeit der Schüler/innen von ihren Lehrpersonen führen (Meloth und Deering, 1999). Interventionen können unter Umständen im Dienste der Selbständigkeit stehen, indem gezielt Lern- und Zusammenarbeitskompetenzen modelliert oder erarbeitet werden, zum Beispiel dann, wenn Lehrpersonen die Kleingruppensituation nutzen, um mit den Schülern und Schülerinnen adäquate Argumentationsmuster und Zusammenarbeitsformen zu erarbeiten (Meloth und Deering, 1999; Cobb, Wood und Yackel, 1993). So gesehen müssen sich die aktiv-interaktive Auseinandersetzung mit dem Lerngegenstand durch autonome Lernende einerseits und die Zusammenarbeit mit der Lehrperson bzw. das Aufnehmen von Steuerungsimpulsen von der Lehrperson andererseits nicht ausschliessen; dies umso weniger, wenn es gelingt, eine Lernkultur aufzubauen, in der sich eine Schulklasse oder Lerngruppe als "Wissensbildungsgemeinschaft» (Brown, 1997) versteht, in der es als «normal» erlebt wird, dass Individuen mit unterschiedlichem Wissensstand in unterschiedlichen Situationen zusammenarbeiten und sich gegenseitig unterstützen. Dieser optimistischen Sicht stehen die Ergebnisse der Mikroanalysen der Arbeitsgruppe um Dann, Diegritz und Rosenbusch (1999) gegenüber, die zeigen, dass Lehrerinterventionen zumindest unter 
gewissen Bedingungen negative Auswirkungen auf den Verlauf der Gruppenarbeit haben können. Die Frage der Lehrerinterventionen bedarf - vor allem vor dem Hintergrund der vielfach beobachteten mangelhaften Qualität von Lerndialogen bei Gruppen- oder Partnerarbeiten - deshalb immer noch der Klärung durch weitere Untersuchungen. So wäre zu prüfen, welche Möglichkeiten es gibt, die Qualität von Lerndialogen zu sichern oder wiederherzustellen, ohne die von Dann et al. beobachteten negativen Nebenwirkungen zu erzeugen. Auch die Frage, ob und unter welchen Bedingungen es sinnvoller ist, dass die Lehrperson bereits während der Gruppen- oder Partnerarbeit in die Lerndialoge eingreift und die Lernenden anleitet, und unter welchen Bedingungen es sinnvoller ist, Missverständnisse und Fehllösungen beispielsweise erst in der Auswertung zu diskutieren, ist noch nicht beantwortet. Um solche Fragen zu klären, sind weitere Analysen von Gruppen- und Partnergesprächen und der darin stattfindenden Lehr-Lerndialoge unter kontrollierten Bedingungen (v.a. des Lehrerverhaltens) notwendig. Sie können in sinnvoller Weise ergänzt werden durch die Beobachtung authentischen Gruppenunterrichts im Rahmen von Felduntersuchungen, wie sie von Hogan, Nastasi und Pressley (1999) und der Forschungsgruppe von Dann, Diegritz und Rosenbusch (1999) praktiziert wurden. Letztere gehen davon aus, dass die Alltagspraxis des Gruppenunterrichts ein Potenzial an von Lehrpersonen für unterschiedlichste Bedürfnisse und Kontexte entwickelten kreativen Lösungen birgt, das von der Unterrichtsforschung noch längst nicht ausgeschöpft ist (Dann, Diegritz und Rosenbusch, 1999, S. 9) - eine Annahme, der wir voll und ganz zustimmen. ${ }^{4}$

\section{Anmerkungen}

1 Bezeichnungen wie kooperatives Lernen, Gruppenunterricht, Gruppenarbeit werden in der Literatur nicht einheitlich verwendet. Kooperatives Lernen oder Gruppenunterricht wird in der Regel als Sammelbegriff für didaktische Methoden verwendet, die als gemeinsames Merkmal die zeitweilige Aufteilung der Klasse in Kleingruppen aufweisen (Cohen, 1994; Dann, Diegritz und Rosenbusch, 1999; Webb und Palincsar, 1996). Die Phasen der Aktivitäten in den Kleingruppen im engeren Sinne werden als Gruppenarbeit bezeichnet. Auch in englischsprachigen Publikationen werden Bezeichnungen für kooperatives Lernen keineswegs einheitlich verwendet. Was genau unter "peer collaboration", "collaborative learning», "cooperative learning», "peer assisted learning», oder "peer learning» verstanden wird, muss daher jedesmal neu festgelegt werden.

2 Es sollte aber nicht übersehen werden, dass vermutlich zwischen Schulstufen, Schultypen und Schulfächern grosse Unterschiede bestehen.

3 Auch Lehrerstudentinnen und -studenten zeigen sich skeptisch, was die Effektivität kooperativer Lernformen betrifft. Dies stellte die Ko-Autorin wiederholt anlässlich der Durchführung von Fachkursen zum kooperativen Lernen in der Lehrerbildung fest.

4 In diesem Sinne werden wir auch unsere eigene Forschungsarbeit im Bereich des kooperativen Lernens im Rahmen einer Video-Studie über Mathematiklernen in den drei grossen Sprachregionen der Schweiz und im Ländervergleich u.a. mit Deutschland fortsetzen (Reusser, Pauli und Zollinger, 1998). 


\section{Literatur}

Aeschbacher, U. (1989). Reziprokes Lehren. Eine amerikanische Unterrichtsmethode zur Verbesserung des Textverstehens. Beiträge zur Lehrerbildung, 7(2), 194-204.

Bauersfeld, H. (1995). "Language games» in the mathematics classrooms: Their function and their effects. In P. Cobb \& H. Bauersfeld (Eds.), The emergence of mathematical meaning: Interaction in classroom cultures (pp. 271-291). Hillsdale, NJ: Erlbaum.

Beaton, A. E., Mullis, I. V. S., Martin, M. O., Gonzales, E. J., Kelly, D. L. \& Smith, T. A. (1996). Mathematics achievement in the middle school years. IEA's Third International Mathematics and Science Study. Chestnut Hill, MA: Boston College.

Berkowitz, M. \& Gibbs, J. (1983). Measuring the developmental features of moral discussion. Merrill-Palmer Quarterly, 29, 399-410.

Brown, A.L. (1992). Design experiments: Theoretical and methodological challenges in creating complex interventions in classroom settings. The Journal of the Learning Sciences, 2, 141-178.

Brown, A.L. (1997). Transforming schools into communities of thinking and learning about serious matters. American Psychologist, 52(4), 399-413.

Cobb, P., Wood, T. \& Yackel, E. (1993). Discourse, mathematical thinking, and classroom practice. In E.A. Forman, N. Minick \& C.A. Stone (Eds.), Contexts for learning. Sociocultural dynamics in children's development (pp. 91-119). New York: Oxford University Press.

Cohen, E.G. (1994). Restructuring the classroom: Conditions for productive small groups. Review of Educational Research, 64(1), 1-35.

Cooper, M.A. (1999). Classroom choices from a cognitive perspective on peer learning. In A.M. O’Donnell \& A. King (Eds.), Cognitive perspectives on peer learning (pp. 213-233). Mahwah, NJ: Erlbaum.

Dann, H.-D., Diegritz, T. \& Rosenbusch, H.S. (Hrsg.). (1999). Gruppenunterricht im Schulalltag. Realität und Chancen. Erlangen: Universitätsbibliothek (= Erlangener Forschungen, Reihe A, Bd. 90).

De Lisi, R. \& Golbeck, S.L. (1999). Implications of Piagetian theory for peer learning. In A.M. O’Donnell \& A. King (Eds.), Cognitive perspectives on peer learning (pp. 3-37). Mahwah, NJ: Erlbaum.

Denecke, W. \& Ritz, E. (1993). Gruppenarbeit im Netz pädagogischer Ansprüche. In H. Gudjons (Hrsg.), Handbuch Gruppenunterricht (S. 138-196). Weinheim; Basel: Beltz.

Diegritz, T., Rosenbusch, H.S. \& Dann, H.-D. (1999). Neue Aspekte einer Didaktik des Gruppenunterrichts. In H.-D. Dann, T. Diegritz \& H.S. Rosenbusch (Hrsg.), Gruppenunterricht im Schulalltag. Realität und Chancen (S. 331-356). Erlangen: Universitätsbibliothek (= Erlangener Forschungen, Reihe A, Bd. 90).

Dillenbourg, P. (Ed. 1999). Collaborative learning. Cognitive and computational approaches. Amsterdam: Pergamon.

Dillenbourg, P. (1999). Introduction: What do you mean by «collaborative learning»? In P. Dillenbourg (Ed.), Collaborative learning. Cognitive and computational approaches (pp. 119). Amsterdam: Pergamon.

Doise, W. \& Mugny, G. (1984). The Social Development of the Intellect. Oxford: Pergamon Press.

Edwards, D. \& Mercer, N. (1989). Reconstructing context: the conventionalization of classroom knowledge. Discourse Processes, 12, 91-104.

Foot, H. \& Howe, C.J. (1998). The psychoeducational basis of peer-assisted learning. In K. Topping \& S. Ehly (Eds.), Peer-assisted learning (pp. 27-43). Mahwah, NJ: Erlbaum.

Forman, E.A. \& McPhail, J. (1993). Vygotskian perspective on children's collaborative problem-solving activities. In E.A. Forman, N. Minick \& C.A. Stone (Eds.), Contexts for learning. Sociocultural dynamics in children's development (pp. 213-229). New York: Oxford University Press. 
Fürst, C. (1999). Die Rolle der Lehrkraft im Gruppenunterricht. In H.-D. Dann, T. Diegritz \& H.S. Rosenbusch (Hrsg.), Gruppenunterricht im Schulalltag. Realität und Chancen (S. 107-150). Erlangen: Universitätsbibliothek (= Erlangener Forschungen, Reihe A, Bd. 90).

Gräsel, C. \& Gruber, H. (2000). Kooperatives Lernen in der Schule. Theoretische Ansätze empirische Befunde - Desiderate für die Lehramtsausbildung. In N. Seibert (Hrsg.), Unterrichtsmethoden kontrovers (S. 161-175). Bad Heilbrunn: Klinkhardt.

Gudjons, H. (1993). Gruppenunterricht. Eine Einführung in Grundfragen. In H. Gudjons (Hrsg.), Handbuch Gruppenunterricht (S. 12-53). Weinheim; Basel: Beltz.

Gudjons, H. (Hrsg.) (1993). Handbuch Gruppenunterricht. Weinheim; Basel: Beltz.

Helmke, A. \& Weinert, F.E. (1997). Unterrichtsqualität und Leistungsentwicklung. In F.E. Weinert \& A. Helmke (Hrsg.), Entwicklung im Grundschulalter (S. 241-252). Weinheim: Psychologie Verlags Union.

Herrenkohl, L.R., Palincsar, A.S., DeWater, L.S. \& Kawasaki, K. (1999). Developing scientific communities in classrooms: A sociocognitive approach. The Journal of the Learning Sciences, $8(384), 451-493$.

Hogan, K., Nastasi, B.K. \& Pressley, M. (1999). Discourse patterns and collaborative scientific reasoning in peer and teacher-guided discussions. Cognition and Instruction, 17(4), 379432.

Hogan, D.M. \& Tudge, J.R.H. (1999). Implications of Vygotskian theory for peer learning. In A.M. O’Donnell \& A. King (Eds.), Cognitive perspectives on peer learning. Mahwah, NJ: Erlbaum.

Huber, G.L. (1987). Kooperatives Lernen: Theoretische und praktische Herausforderung für die Pädagogische Psychologie. Zeitschrift für Entwicklungspsychologie und Pädagogische Psychologie, 19(4), 340-362.

Huber, G.L. (1991). Methoden des kooperativen Lernens. In E. Meyer \& R. Winkel (Hrsg.), Unser Konzept: Lernen in Gruppen (S. 166-174). Hohengehren: Schneider.

Huber, G.L. (1994). Lernprozesse in Kleingruppen: Wie kooperieren Lerner? Unterrichtswissenschaft, 23(4), 316-331.

IAEP (1992). Learning mathematics. Princeton, NJ: Educational testing service.

Järvelä, S. (1996). Qualitative features of teacher-student interaction in a technologically rich learning environment based on a cognitive apprenticeship model. Machine-Mediated Learning, 5(2), 91-107.

Johnson, D.W. \& Johnson, R.T. (1992a). Encouraging thinking through constructive controversy. In N. Davidson \& T. Worsham (Eds.), Enhancing thinking through cooperative learning (pp.120-137). New York: Teachers College Press.

Johnson, D.W. \& Johnson, R.T. (1992b). Positive interdependence: Key to effective cooperation. In R. Hertz-Lazarowitz \& N. Miller (Eds.), Interaction in cooperative groups. The theoretical anatomy of group learning (pp. 174-199). Cambridge: Cambridge Univesity Press.

King, A. (1994). Guiding knowledge construction in the classroom: Effects of teaching children how to question and how to explain. American Educational Research Journal, 31(2), 336-368.

King, A. (1999). Discourse patterns for mediating peer learning. In A.M. O’Donnell \& A. King (Eds.), Cognitive perspectives on peer learning (pp. 87-115). Mahwah, NJ: Erlbaum.

Krapf, B. (1994). Aufbruch zu einer neuen Lernkultur. Erhebungen, Experimente, Analysen und Berichte zu pädagogischen Denkfiguren (3.Aufl.). Bern: Haupt.

Lampert, M. (1990). When the problem is not the question and the solution is not the answer: Mathematical knowing and teaching. American Educational Research Journal, 27, $29-63$.

Lemke, J.L. (1990). Talking science: Language, learning and values. Norwood, N.J.: Ablex.

Littleton, K. \& Häkkinen, P. (1999). Learning together: Understanding the processes of computer-based collaborative learning. In P. Dillenbourg (Eds.), Collaborative learning. Cognitive and computational approaches (pp. 20-30). Amsterdam: Pergamon. 
Maheady, L. (1998). Advantages and disadvantages of peer-assisted learning strategies. In K. Topping \& S. Ehly (Eds.), Peer-assisted learning (pp. 45-65). Mahwah, NJ: Erlbaum.

Mehan, H. (1979). Learning lessons. Social organization in the classroom. Cambridge, MA: Harward University Press.

Meloth, M.S. \& Deering, P.D. (1999). The role of the teacher in promoting cognitive processing during collaborative learning. In A.M. O’Donnell \& A. King (Eds.), Cognitive perspectives on peer learning (pp. 235-255). Mahwah, NJ: Erlbaum.

Mercer, N. (1995). The guided construction of knowledge. Talk amongst teachers and learners. Clevedon: Multilingual Matters.

Meyer, E. (1996). Gruppenunterricht - Grundlegung und Beispiel (9. Aufl.). Oberursel: Wunderlich (Original 1954).

Meyer, H. (1987). Unterrichtsmethoden II: Praxisband (3. Aufl.). Frankfurt a.M.: Cornelsen Scriptor.

Nuhn, H.-C. (1995). Partnerarbeit als Sozialform des Unterrichts. Weinheim: Beltz.

O’Donnell, A.M. \& Dansereau, D.F. (1992). Scripted cooperation in student dyads: a method for analyzing and enhancing academic learning and performance. In R.Hertz-Lazarowitz \& N. Miller (Eds.), Interaction in cooperative groups. The theoretical anatomy of group learning (pp. 120-141). Cambridge: Cambridge University Press.

O’Donnell, A.M. \& King, A. (1999). Cognitive perspectives on peer learning. Mahwah, NJ: Erlbaum.

Palincsar, A., \& Brown, A.L. (1984). Reciprocal teaching and comprehension-fostering and comprehension-monitoring activities. Cognition and Instruction, 1(2), 117-175.

Pauli, C. (1998). Computerunterstützte Schülerzusammenarbeit im Mathematikunterricht. Unveröffentlichte Dissertation, Universität Zürich: Pädagogisches Institut.

Perret-Clermont, A.-N. (1980). Social interaction and cognitive development in children. London: Academic Press.

Piaget, J. (1947/dt. 1971). Psychologie der Intelligenz. Olten: Walter.

Renkl, A. \& Mandl, H. (1995). Kooperatives Lernen: Die Frage nach dem Notwendigen und dem Ersetzbaren. Unterrichtswissenschaft, 23(4), 292-300.

Reusser, K. (1993). Tutoring systems and pedagogical theory: Representational tools for understanding, planning, and reflection in problem-solving. In S.P. Lajoie \& S. Derry (Eds.), Computers as cognitive tools (pp. 143-177). Hillsdale, NJ: Erlbaum.

Reusser, K. (2000, in press). Co-constructivism in educational theory and practice. In N.J. Smelser, P. Baltes \& F.E. Weinert (Eds.), International Encyclopedia of the Social and Behavioral Sciences. Oxford: Pergamon/Elsevier Science.

Reusser, K., Pauli, C. \& Zollinger, A. (1998). Mathematiklernen in verschiedenen Unterrichtskulturen - eine Videostudie im Anschluss an TIMSS. Beiträge zur Lehrerbildung, 16(3), 427-438.

Roschelle, J. \& Teasley, S.D. (1995). The construction of shared knowledge in collaborative problem solving. In C. O’Malley (Ed.), Computer supported collaborative learning (pp. 6997). Berlin; New York u.a.: Springer.

Rogoff, B. (1990). Apprenticeship in thinking. Cognitive Development in social context. New York; Oxford: Oxford University Press.

Rosenshine, B. \& Meister, C. (1994). Reciprocal teaching: A review of the research. Review of Educational Research, 64(4), 479-530.

Slavin, R.E. (1993). Kooperatives Lernen und Leistung: Eine empirisch fundierte Theorie. In G.L. Huber (Hrsg.), Neue Perspektiven der Kooperation. Ausgewählte Beiträge der Internationalen Konferenz 1992 über Kooperatives Lernen (= Grundlagen der Schulpädagogik, Bd.6; S. 151-170). Baltmannsweiler: Schneider.

Stebler, R. (1999). Eigenständiges Problemlösen. Zum Umgang mit Schwierigkeiten beim individuellen und paarweisen Lösen mathematischer Problemgeschichten. Bern: Lang. 
Stebler, R. \& Reusser, K. (1996). Learning together: When good students underestimate the benefits of peer collaboration. Paper presented at the congress «The Growing Mind. Multidisciplinary Approaches». September 1996, Geneva.

Stebler, R. \& Reusser, K. (2000). Progressive, classical, or balanced - A look at mathematical learning environments in Swiss-German lower-secondary schools. Zentralblatt für Didaktik der Mathematik (ZDM), 32(1), 1-10.

Topping, K. \& Ehly, S. (Eds.) (1998). Peer-assisted learning. Mahwah, NJ: Erlbaum.

Tudge, J. (1989). When collaboration leads to regression: some negative consequences of sociocognitive conflict. European Journal of Social Psychology, 19, 123-138.

Vettiger, H. (1987). Arbeit in Kleingruppen. In H. Messner (Hrsg.), Unterrichten lernen (S. 139-161). Hannover: Schroedel.

Von Hanffstengel, U. (1999). Innere Konflikte der Lehrkräfte im Gruppenunterricht. In H.-D. Dann, T. Diegritz \& H.S. Rosenbusch (Hrsg.), Gruppenunterricht im Schulalltag. Realität und Chancen (S. 273-300). Erlangen: Universitätsbibliothek (= Erlangener Forschungen, Reihe A, Bd. 90).

Vygotsky, L.S. (1978). Mind in society. The development of higher psychological processes. Cambridge, MA: Harvard University Press.

Webb, N.M. \& Palincsar, A. S. (1996). Group processes in the classroom. In D.C. Berliner \& R.C. Calfee (Eds.), Handbook of Educational Psychology (pp. 841-873). New York: MacMillan.

Weinert, F.E. (1997). Lernkultur im Wandel. In E. Beck, T. Guldimann \& M. Zutavern (Hrsg.), Lernkultur im Wandel (S. 10-29). St. Gallen: UVK

\section{Du rôle de l'enseignant lors de l'apprentissage coopératif}

\section{Résumé}

Malgré l'existence d'une importante littérature de recherche sur l'apprentissage coopératif et indépendant, peu de publications ont porté sur le rôle et l'efficacité du maître lors de situations de travail à deux ou en groupes sous l'angle de l'initiation et de l'accompagnement des dialogues entre élèves. Mais il est connu que tous les dialogues et interactions qui se développent dans des situations coopératives ne présentent pas les caractéristiques de qualité à partir desquels les pédagogues et enseignants aiment à travailler. Sur la base d'hypothèses explicatives courantes sur l'usage des formes d'enseignement et d'apprentissage coopératifs, le présent article discute de la qualité des dialogues d'apprentissage dans le travail de groupe ou en dyade à partir de plusieurs critères. L'article aborde ensuite la fonction des enseignants dans l'obtention de cette qualité. Finalement, des conclusions sont tirées quant à l'orientation de la recherche sur le rôle de l'enseignant et de l'accompagnement dans des situations d'enseignement dialogique. 


\title{
II ruolo dell'insegnante nell'apprendimento cooperativo
}

\author{
Riassunto
}

Nonostante sia disponibile un'ampia letteratura sul tema dell'apprendimento cooperativo e autonomo, poche sono le pubblicazioni che affrontano il problema dell'efficacia dell'insegnante nell'ambito di attività di gruppo e a coppie e nello stimolare dialoghi di apprendimento. Per contro è risaputo che i dialoghi e le interazioni che si producono in contesti di apprendimento cooperativo non rispondono alle caratteristiche qualitative volentieri presupposte da pedagogisti e insegnanti. Partendo da relative ipotesi sull'utilità di forme di apprendimento cooperative, in questo contributo si discute la qualità dei dialoghi in relazione a prestazioni multicriteriali. Di seguito si approfondiscono le funzioni dell'insegnante per rapporto al raggiungimento di tale qualità e si forniscono indicazioni per l'orientamento della ricerca sul ruolo dell'insegnante e sull'accompagnamento delle forme di apprendimento dialogico.

\section{To the Role of the Teacher in Cooperative Learning}

\section{Summary}

In spite of the presence of volumes of research literature about cooperative and independent learning, there are only a few publications regarding the role and effectiveness of the teacher in pair and group work respective to the initiation and accompaniment of student-active educational dialogues. On the other hand, it is known that not all the dialogues and interactions that take place in cooperative arrangements show the quality features that pedagogues and teachers like to proceed from. On the basis of current explanatory hypotheses on the use of cooperative teaching and learning models, the following article discusses the quality of dialogues for a multi-critical procedure of partner- and group work. Subsequently, it addresses the function of teachers regarding the obtainment of this quality. Finally, conclusions are drawn as to the orientation of research into the teacher and learner's roll that accompanies dialogical teaching. 